\title{
Efecto de la suplementación dietética con harina de morera (Morus alba) sobre el desempeño productivo de codornices (Coturnix coturnix japonica) en crecimiento
}

\author{
Effect of dietary supplementation with mulberry (Morus alba) meal on the \\ productive performance of growing quails (Coturnix coturnix japonica)
}

Daniel A. Perdomo C. ${ }^{1,2}$, Ana Briceño ${ }^{2}$, Doraida Díaz C..$^{1,2}$, Diomary González ${ }^{1,2}$, Líber González ${ }^{2}$, Pedro A. Moratinos L. ${ }^{1,2}$, Eliani K. Núñez G. ${ }^{2}$, Fernando P. Perea G. ${ }^{1,2,3,4}$

\section{Resumen}

\begin{abstract}
El estudio tuvo como objetivo evaluar el comportamiento productivo de codornices de engorde (Coturnix coturnix japonica) alimentadas con alimento balanceado y suplementadas con harina de morera (Morus alba). El experimento se desarrolló en el Estado Trujillo, Venezuela, durante seis semanas. Se aplicó un diseño completamente aleatorizado con tres tratamientos (dietas) y cinco repeticiones por tratamiento (4 aves por repetición). Las dietas fueron: T0: 100\% alimento balanceado (AB) comercial; T1: $90 \% \mathrm{AB}+$ $10 \%$ de harina de morera $(\mathrm{HM}) ; \mathrm{T} 2: 80 \% \mathrm{AB}+20 \% \mathrm{HM}$. Se determinó el peso final $(\mathrm{PF})$, ganancia diaria de peso (GDP), consumo de alimento, tasa de crecimiento específica (TCE), conversión alimenticia (CA), rendimiento en canal (RC), mortalidad y relación costo beneficio (CB). Los datos fueron procesados mediante el análisis de varianza usando el procedimiento GLM del programa SAS. El PF y la GDP fueron mayores en T1 y T2 (PF: T0: $139.1 \pm 1.9 ;$ T1: 147.2 $\pm 2.0 ;$ T2: $147.1 \pm 1.9$ (p<0.03)(GDP: T0: $3.1 \pm 0.05 ;$ T1: 3.3 $\pm 0.05 ; \mathrm{T} 2: 3.3 \pm 0.05(\mathrm{p}<0.03)$. Las aves de $\mathrm{T} 1$ y T2 consumieron más alimento que las de T0 $(p<0.01)$. La TCE fue menor $(p<0.05)$ en T0 (7.01\%) que en T1 (7.13\%) y T2 (7.14\%). La $\mathrm{CA}$ y el RC no variaron entre tratamientos. La relación CB favoreció a las aves de T1 y T2. Se concluye que la adición de HM en la ración mejoró el PF, la GDP y la relación CB, constituyendo una alternativa para reducir los costos de alimentación de los establecimientos comerciales, sin menoscabo de los índices productivos de las aves.
\end{abstract}

Palabras clave: codornices; dieta; Morus alba; crecimiento; relación costo beneficio

\footnotetext{
${ }^{1}$ Grupo de Investigación en Producción Animal (GIPA), Núcleo Universitario «Rafael Rangel», Universidad de Los Andes, Trujillo, Venezuela

${ }^{2}$ Departamento de Ciencias Agrarias, Núcleo Universitario «Rafael Rangel», Universidad de Los Andes, Trujillo, Venezuela

${ }^{3}$ Facultad de Ciencias Agropecuarias, Universidad de Cuenca, Ecuador

${ }^{4}$ E-mail:ferromi9@gmail.com
} 
The aim of this study was to evaluate the productive performance of growing quail (Coturnix coturnix japonica) fed with a commercial balanced feed and supplemented with mulberry meal (Morus alba). The experiment was carried out in Trujillo, Venezuela, for six weeks. A completely randomized design was applied with three treatments (diets) and five repetitions per treatment (4 birds per repetition). The diets were: T0: 100\% commercially balanced feed (AB); $\mathrm{T} 1: 90 \% \mathrm{AB}+10 \%$ of mulberry meal (HM); $\mathrm{T} 2: 80 \% \mathrm{AB}$ $+20 \%$ HM. The final body weight (FW), daily weight gain (DWG), feed consumption (DI), specific growth rate (SGR), feed conversion (FC), carcass yield (CY), mortality and cost-benefit ratio were determined $(\mathrm{CB})$. The data were processed by analysis of variance using the GLM procedure of the SAS program. The FW and the DWG were higher in T1 and T2 (PF: T0: $139.1 \pm 1.9$, T1: 147.2 $\pm 2.0, \mathrm{~T} 2: 147.1 \pm 1.9,(\mathrm{p}<0.03)$ (DWG: T0: $3.1 \pm 0.05$, $\mathrm{T} 1: 3.3 \pm 0.05 ; \mathrm{T} 2: 3.3 \pm 0.05(\mathrm{p}<0.03)$. The birds of $\mathrm{T} 1$ and $\mathrm{T} 2$ consumed more feed than those of T0 $(\mathrm{p}<0.01)$. The SGR was lower $(\mathrm{p}<0.05)$ in T0 $(7.01 \%)$ than in T1 $(7.13 \%)$ and T2 (7.14\%). FC and CY did not vary between treatments. The CB ratio favored the birds of T1 and T2. It is concluded that the addition of HM in the ration improved FW, DWG and CB, constituting an alternative to reduce the feeding costs of the commercial farms without diminishing the productive indices of the birds.

Key words: quails; diet; Morus alba; growth; cost-benefit ratio

\section{INTRODUCCIÓN}

El consumo de fuentes alternativas de carne, como codorniz, viene cobrando importancia en Venezuela, debido a la escasez de las fuentes tradicionales. La carne de codorniz presenta algunas cualidades que la hacen superior a otras especies avícolas, tales como la terneza, jugosidad, buen sabor y bajo conteni do de grasa(Dal mau, 1994; Lázaro et al., 2005; Genchev et al., 2008). Estas aves muestran un crecimiento precoz, lo que reduce el periodo de engorde, alcanzando el peso vivo adulto antes que otras especies avícolas como el pollo o el pavo. Además, también tienen la particularidad de lograr una madurez sexual temprana, y por ser especie polígama posee importantes diferencias morfológicas entre sexos (Santomá, 1989; Lázaro et al., 2005).

Particularmente en Venezuela, la crianza de codornices se ha orientado principalmente hacia la producción de huevos, mientras que los machos se les comercializa como carne (Cori et al., 2009). En la mayoría de los casos la explotación comercial de la codorniz es fundamentalmente intensiva, y sus productos gozan de una imagen más natural que el de otras aves, lo cual la hace muy atractiva para el consumidor (Lázaro et al., 2005).

Los países en vía de desarrollo han adoptado tecnologías de los países desarrollados en los cuales la producción de alimentos para los animales de granja se realiza a base de granos y oleaginosas que en muchos casos encarecen el costo del alimento. Una alternativa que viene siendo empleada es la orientación de los sistemas de producción hacia modelos más sustentables, empleando otras fuentes alimenticias, como la harina de la hoja de la morera, Morus alba (Itza-Ortiz et al., 2010). Asimismo, se han utilizado la palma africana (Morantes, 1995), la semilla de girasol (Arslan et al., 2001), la harina de maní (Bayram y Akinci, 2001), la harina de batata (Real, 1996) y la harina de lombriz (Díaz et al., 2007, 2009). Estas referencias indican el interés en probar fuentes alimenticias dife- 
rentes a las que usualmente se han usado en los establecimientos comerciales, con el fin de disminuir los costos de la alimentación convencional (Ustundag y Ozdogan, 2015). Se considera que, aun hoy en día, la información estandarizada sobre los requerimientos nutricionales de las codornices es escasa y poco actualizada, siendo las recomendaciones del NRC las adoptadas para la formulación de las dietas (NRC, 1994).

Se considera que la morera es una fuente alimenticia de alto valor nutritivo y con una excelente composición química (Benavides, 2002; Ustundag y Ozdogan, 2015). Esta planta exhibe un elevado contenido de proteína bruta, además de una destacada producción de biomasa cuando es manejada apropiadamente, razón por la cual se ha utilizado en múltiples investigaciones con la finalidad de evaluar su potencial nutricional. Entre las especies animales en las que se ha usado se destacan los pollos de engorde (Casamachin et al., 2007; Itzá-Ortiz et al., 2010), rumiantes (Benavides, 2002) y conejos (Nieves et al., 2004; Martínez et al., 2005).

En base a estos antecedentes, se estableció como objetivo del presente estudio evaluar el efecto de la suplementación dietética de la harina de morera (Morus alba) sobre el comportamiento productivo de codornices en crecimiento (Coturnix coturnix japonica).

\section{Materiales y Métodos}

\section{Ubicación del Ensayo}

El estudio se desarrolló en el Laboratorio de Avicultura del Núcleo Universitario «Rafael Rangel» (NURR) de la Universidad de Los Andes (ULA), Estado Trujillo, Venezuela, el cual se localiza a $419 \mathrm{msnm}$, en un área de bosque seco tropical, con temperatura promedio de $27.4{ }^{\circ} \mathrm{C}$, precipitación anual de $1328 \mathrm{~mm}$ y una humedad relativa de $71 \%$ (Díaz et al., 2009).

\section{Manejo de las Aves}

La prueba de alimentación se realizó durante un periodo de seis semanas. Se trabajó con 60 codornices Coturnix coturnix japonica sin sexar de un día de edad. Se emplearon 15 jaulas $(32 \times 50 \times 30 \mathrm{~cm})$, con comederos y bebederos individuales. Las aves se distribuyeron al azar, en tres grupos o tratamientos $(\mathrm{T})$ con cinco repeticiones por $\mathrm{T}$ $(n=20$ por tratamiento). El peso vivo promedio inicial fue de T0: $7.3 \pm 0.2 \mathrm{~g}, \mathrm{~T} 1: 7.4 \pm 0.2$ g y T2: $7.1 \pm 0.2 \mathrm{~g}$.

Las codornices fueron pesadas semanalmente para determinar la ganancia de peso, con una balanza semi-analítica Ohaus SP 402 (EEUU) con precisión de 0.01 g. El consumo de alimento por animal fue evaluado diariamente y se calculó mediante la diferencia entre el alimento suministrado y el remanente en el comedero, mediante una balanza digital US-Bench Top-PRO con precisión de $0.1 \mathrm{~g}$ (US Balance, EEUU).

\section{Dietas}

Se utilizó una dieta comercial para pollos de engorde (Pollarina P-1 ${ }^{\circledR}$, Concentrados Valera) con $17.5 \%$ de proteína (T0: $100 \%$ $\mathrm{AB})$. En las dietas T1 y T2 se sustituyó 10 y $20 \%$ de la dieta comercial por harina de morera (HM), respectivamente. Las dietas fueron suministradas ad libitum y en forma de harina.

Las hojas de morera para la elaboración de la harina fueron recolectadas en la Finca Dorana, ubicada en el sector El Say, del municipio Boconó, Trujillo, a una altura de 1960 msnm, y con temperatura promedio de $17^{\circ} \mathrm{C}$. Se seleccionó una planta de 5 años y se realizaron cortes al azar en las diferentes ramas. Las hojas fueron retiradas de las ramas, y posteriormente cortadas, deshidratadas, molidas y analizadas. La deshidratación se efectuó en una estufa a $60{ }^{\circ} \mathrm{C}$ durante 48 horas, la molienda se hizo en una licuadora y el material molido (aproximadamente $10 \mathrm{~kg}$ ) se almacenó en un envase hermético. 
Cuadro 1. Análisis bromatológico y energía metabolizable de la harina de morera (Morus $a l b a$ ) y de las tres dietas experimentales (en base seca $\mathrm{g} / 100$ )

\begin{tabular}{cccccccccc}
\hline Muestra & $\begin{array}{c}\text { MS } \\
(\%)\end{array}$ & $\begin{array}{c}\mathrm{H} \\
(\%)\end{array}$ & $\begin{array}{c}\text { CEN } \\
(\%)\end{array}$ & $\begin{array}{c}\text { MO } \\
(\%)\end{array}$ & $\begin{array}{c}\text { PC } \\
(\%)\end{array}$ & $\begin{array}{c}\text { EE } \\
(\%)\end{array}$ & $\begin{array}{c}\text { FC } \\
(\%)\end{array}$ & $\begin{array}{c}\text { ELN } \\
(\%)\end{array}$ & $\begin{array}{c}\text { EM } \\
\mathrm{kcal} / \mathrm{kg}\end{array}$ \\
\hline HM & 94.16 & 5.84 & 12.3 & 84.2 & 18.3 & 5.12 & 22.4 & 36.0 & 2634.4 \\
T0 & 94.12 & 5.88 & 10.8 & 89.2 & 17.5 & 5.81 & 3.9 & 56.1 & 3467.4 \\
T1 & 93.58 & 6.42 & 11.7 & 88.3 & 17.2 & 5.08 & 7.5 & 52.1 & 3229.2 \\
T2 & 93.72 & 6.28 & 11.9 & 88.1 & 17.4 & 5.28 & 9.5 & 49.6 & 3155.2 \\
\hline
\end{tabular}

HM: harina de morera; T0: Dieta comercial (DC); T1: DC con 10\% HM; T2: DC con 20\% HM

MS: Materia Seca; H: Humedad; CEN: Cenizas; MO: Materia Orgánica; PC: Proteína Cruda; EE: Extracto

Etéreo; FC: Fibra Cruda; ELN: Extracto Libre de Nitrógeno; EM: Energía metabolizable

La composición bromatológica de cada dieta y de la HM se realizó según la metodología estandarizada de la AOAC (1995) en el Laboratorio de Química Ambiental (LAQUIAM) del NURR-ULA. La energía metabolizable de la HM y de las tres raciones fue calculada de forma indirecta mediante los factores Artwater (Hodgkinson et al., 2004; Díaz et al., 2007, 2009), procurando asegurar que las dietas experimentales mantuvieran condiciones isocalóricas (Cuadro 1).

\section{Sacrificio de las Aves}

Al final del ensayo (42 días), las aves fueron sacrificadas siguiendo protocolos usuales (Gaceta Oficial de Venezuela, 2000; Cori et al., 2009; Díaz et al., 2009) para determinar el rendimiento en canal. Para ello, las aves se sometieron a ayuno por 12 horas y solo se aportó agua ad libitum. Las aves fueron sometidas a un aturdimiento eléctrico previo al desangrado. Luego fueron degolladas y desangradas (4-5 min), se sumergieron en baño de agua $\left(53{ }^{\circ} \mathrm{C}\right)$ para su escaldado por $2 \mathrm{~min}$. Las plumas se retiraron manualmente. Luego se realizó un lavado de las aves y se procedió al corte de patas, cabezas y al eviscerado, todo ello manualmente. En el estudio solo se valoró la canal caliente.

\section{Diseño Experimental y Análisis Estadístico}

Se utilizó un diseño completamente aleatorizado con tres tratamientos cada uno $\mathrm{y}$ con cinco repeticiones por tratamiento (cada una en una jaula). Cada repetición fue considerada una unidad experimental conformada por cuatro codornices. Los animales fueron asignados al azar a cada tratamiento y repetición. El tamaño mínimo muestral se determinó por el método probabilístico simple, aplicándose la fórmula para estimar la media de una variable (Segura y Honhold, 2000).

Los parámetros del comportamiento productivo y crecimiento de las aves se evaluaron mediante las siguientes variables (Sulca et al., 2000; Nieves et al., 2004; Caravaca et al., 2005; Díaz et al., 2009; Itzá-Ortiz et al., 2010; Vatsalya y Arora, 2011):

- Conversión alimenticia (CA): gramos de alimento consumido dividido entre los gramos de incremento de peso vivo.

- Ganancia diaria de peso (GDP): peso final (PF) menos el peso inicial (PI), dividido entre los días del periodo experimental.

- Tasa de crecimiento específica (TCE): logaritmo del PF menos el logaritmo del PI dividido entre el periodo experimental (días), multiplicado por 100. 
Cuadro 2. Parámetros productivos de codornices en crecimiento suplementadas con harina de morera (Morus alba)

\begin{tabular}{lcccc}
\hline Parámetro productivo & T0 & T1 & T2 & P \\
\hline Peso inicial $(\mathrm{g})$ & $7.3 \pm 0.2$ & $7.4 \pm 0.2$ & $7.1 \pm 0.2$ & $\mathrm{Ns}$ \\
Peso final $(\mathrm{g})$ & $139.1 \pm 1.9^{\mathrm{b}}$ & $147.2 \pm 2.0^{\mathrm{a}}$ & $147.1 \pm 1.9^{\mathrm{a}}$ & $<0.03$ \\
Conversión alimenticia & $2.9 \pm 0.06^{\mathrm{a}}$ & $3.8 \pm 0.06^{\mathrm{b}}$ & $4.2 \pm 0.06^{\mathrm{c}}$ & $<0.01$ \\
Ganancia diaria de peso (g/d) & $3.1 \pm 0.05^{\mathrm{b}}$ & $3.3 \pm 0.05^{\mathrm{a}}$ & $3.3 \pm 0.05^{\mathrm{a}}$ & $<0.03$ \\
Rendimiento en canal (\%) & $76.4 \pm 0.72$ & $75.4 \pm 0.76$ & $74.5 \pm 0.75$ & $\mathrm{Ns}$ \\
Mortalidad (\%) & 0 & 0.4 & 0.2 & $\mathrm{Ns}$ \\
Relación costo beneficio & $1.60 \pm 0.02^{\mathrm{a}}$ & $1.30 \pm 0.02^{\mathrm{b}}$ & $1.25 \pm 0.02^{\mathrm{b}}$ & 0.01 \\
\hline
\end{tabular}

T0: Dieta comercial (DC); T1: DC con 10\% HM; T2: DC con 20\% HM

Ns: no significativo

- Rendimiento en canal (RC): peso de la canal dividido entre el peso vivo, multiplicado por 100 .

- Mortalidad (M): número de aves muertas durante el periodo experimental dividido entre las aves vivas al inicio del ensayo, multiplicado por 100 .

- Relación beneficio-costo (CB): se calculó utilizando la fórmula $\mathrm{CB}=[(\mathrm{IPxPC}) /$ (CAxPA)], donde IP: incremento de peso vivo durante el ensayo (g/codorniz), PC: precio de la codorniz viva, CA: consumo neto de alimento durante el ensayo ( $\mathrm{g} / \mathrm{co}-$ dorniz) y PA: precio del alimento.

El efecto de los tratamientos, repeticiones e interacción sobre las variables productivas fue evaluado mediante el análisis de varianza aplicando el procedimiento GLM (Modelo Lineal General) del paquete estadístico SAS (2012). El peso inicial se incluyó como covariable. Las diferencias entre medias fueron analizadas mediante el procedimiento LSMeans del SAS. Se consideran diferencias significativas cuando la probabilidad fue menor a 0.05 .

\section{Resultados y Discusión}

La suplementación con HM influenció los parámetros evaluados (Cuadro 2). Los pesos de las codornices al inicio del experimento fueron similares entre los grupos experimentales y estuvieron dentro de lo reportado por Lázaro et al. (2005), con valores que variaron entre 6 y $9 \mathrm{~g}$ por ave. El PF ajustado por el peso inicial en el análisis de varianza indica que las codornices de $\mathrm{T} 1 \mathrm{y}$ $\mathrm{T} 2$ fueron más pesadas que las de T0 $(p<0.03)$, pero sin diferencias significativas entre T1 y T2. Esto indica que la inclusión del 10 y $20 \%$ de HM produjo un mejor desempeño productivo de las codornices, en comparación con el grupo control; aún a pesar de que los tres grupos fueron alimentados $a d$ libitum. Indistintamente de las dietas usadas en el presente ensayo, el PF de los grupos fue superior a los $127 \mathrm{~g} /$ ave obtenido por Cori et al. (2005) empleando una dieta comercial, pero distan de los valores del peso adulto, que es de 160-250 g según Santomá (1989) o de 225-235 g según Lázaro et al. (2005). 


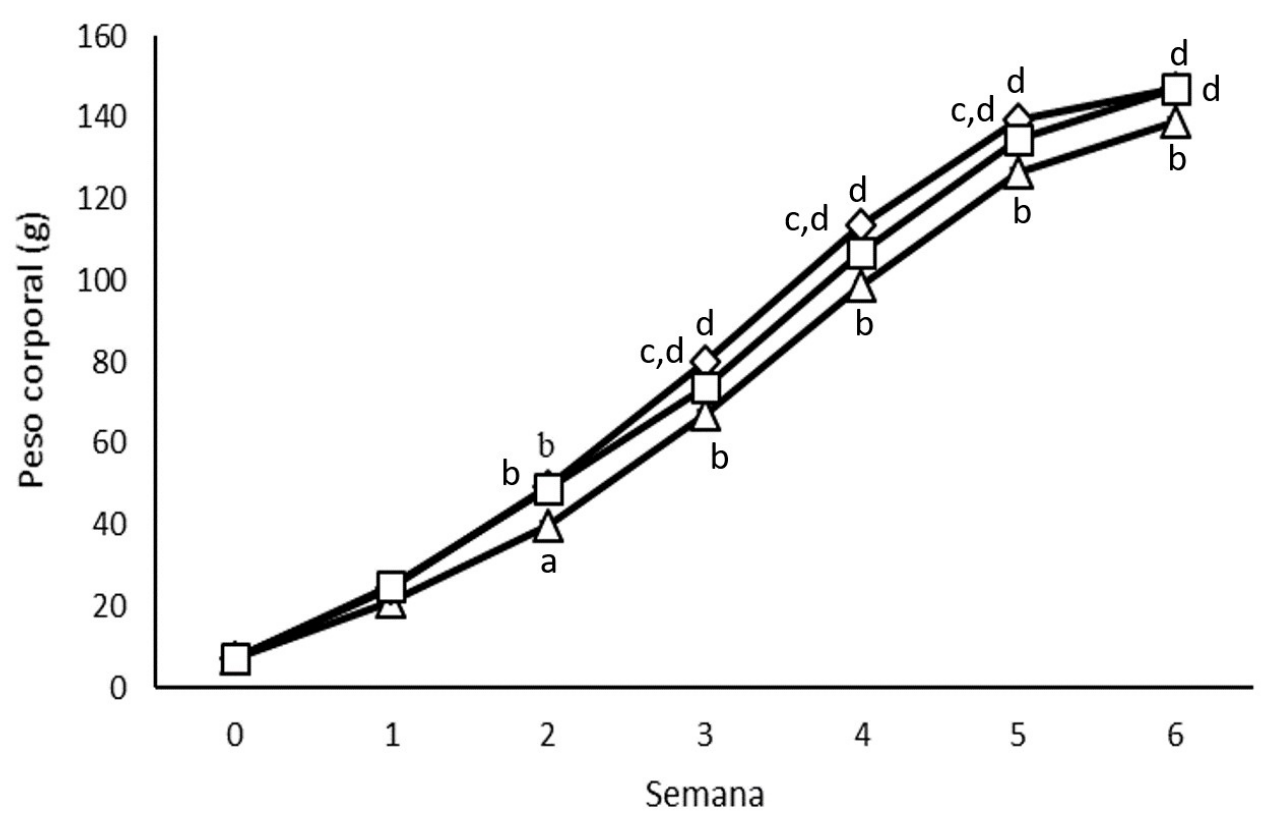

Figura 1. Progresión del peso corporal de codornices (Coturnix coturnix japonica) suplementadas con harina de morera (HM) durante 42 días. T0 (0\% HM): $\Delta$; T1 $(10 \%$ $\mathrm{HM}): \checkmark ; \mathrm{T} 2(20 \% \mathrm{HM}):-$. Letras diferentes en la misma semana difieren ${ }^{\mathrm{a}, \mathrm{b}} \mathrm{p}<0.01$; ${ }^{b, d} \mathrm{p}<0.01 ;{ }^{b, c} \mathrm{p}<0.05$

Las dietas con HM propiciaron un crecimiento continuo ascendente en las codornices, pese a que se ha documentado que niveles crecientes de morera pueden generar un crecimiento decreciente (Panja, 2013). Como muestra la Figura 1, los pesos de las aves fueron proporcionalmente similares durante los primeros siete días del experimento, para luego mejorar en los grupos suplementados con HM con relación al grupo control (dieta comercial). La dieta control mostró durante toda la evaluación un patrón de crecimiento similar al de las dietas de T1 y T2, aunque menos pronunciado.

Estudios en pollos camperos han dado resultados igualmente prometedores al incluirse $10 \%$ de HM en la dieta (Leyva et al., 2012). Islam et al. (2014) observaron que esta suplementación no solo mejoró el desempeño productivo, sino que además causó un descenso en los niveles séricos del colesterol y los triglicéridos. Contrariamente, Panja (2012), al incluir niveles de 0.5 hasta
2\% e Itzá-Ortiz et al. (2010) con inclusión de $4 \%$ de $\mathrm{HM}$ en la dieta no encontraron diferencias significativas en los pesos de pollos de engorde. Asimismo, experiencias llevadas a cabo en gallina ponederas demostraron que la inclusión de 5 y $10 \%$ de morera tampoco afectó la producción de huevos (Camacaro et al., 2015), por lo que su utilización en la elaboración de alimentos para gallinas ponedoras resultó productivamente viable.

La GDP en los tratamientos a base de HM resultaron estadísticamente superiores a la dieta comercial ( $\mathrm{p}<0.05$; Cuadro 2 ). Aun cuando se ha recomendado que $C$. coturnix japonica requiere niveles proteicos entre 24 y $27 \%$ de PB durante las primeras tres semanas de vida, y entre 17 y $22 \%$ desde la $3^{\text {era }}$ semana en adelante (Santomá, 1989; Lázaro et al., 2005), las dietas experimentales demostraron que a pesar de poseer niveles proteicos menores a los sugeridos por estos autores, produjeron un crecimiento favorable durante todo el periodo de engorde de las aves. 
Cuadro 3. Consumo de alimento semanal y total en codornices Coturnix coturnix japonica suplementadas con harina de morera (Morus alba) durante seis semanas

\begin{tabular}{cccccccc}
\hline & \multicolumn{6}{c}{ Semana } & \multirow{2}{*}{ Total } \\
\cline { 2 - 7 } & 1 & 2 & 3 & 4 & 5 & 6 & \\
\hline T0 & $23.4 \pm 2.2^{\mathrm{b}}$ & $47.7 \pm 2.5^{\mathrm{b}}$ & $66.0 \pm 3.6^{\mathrm{b}}$ & $84.9 \pm 2.7^{\mathrm{b}}$ & $94.8 \pm 2.4^{\mathrm{b}}$ & $95.0 \pm 2.2^{\mathrm{c}}$ & $411.6 \pm 9.6^{\mathrm{a}}$ \\
T1 & $35.1 \pm 2.3^{\mathrm{a}}$ & $69.5 \pm 2.6^{\mathrm{a}}$ & $92.7 \pm 3.7^{\mathrm{a}}$ & $112.0 \pm 2.8^{\mathrm{a}}$ & $125.9 \pm 2.5^{\mathrm{a}}$ & $125.4 \pm 2.2^{\mathrm{b}}$ & $556.7 \pm 10.1^{\mathrm{b}}$ \\
T2 & $38.2 \pm 2.2^{\mathrm{a}}$ & $82.3 \pm 2.5^{\mathrm{a}}$ & $105.5 \pm 3.6^{\mathrm{a}}$ & $124.7 \pm 2.7^{\mathrm{a}}$ & $135.5 \pm 2.4^{\mathrm{a}}$ & $137.4 \pm 2.2^{\mathrm{a}}$ & $628.6 \pm 9.9^{\mathrm{c}}$ \\
\hline$p$ & $<0.01$ & $<0.001$ & $<0.01$ & $<0.001$ & $<0.0001$ & $<0.0001$ & $<0.01$ \\
\hline
\end{tabular}

T0: Dieta comercial (DC); T1: DC con 10\% HM; T2: DC con 20\% HM

Cuadro 4. Conversión alimenticia semanal acumulada en codornices Coturnix coturnix japonica suplementadas con harina de morera (Morus alba) durante seis semanas

\begin{tabular}{ccccccc}
\hline & \multicolumn{6}{c}{ Semana } \\
\cline { 2 - 7 } & 1 & 2 & 3 & 4 & 5 & 6 \\
\hline T0 & $1.1 \pm 0.03^{\mathrm{a}}$ & $1.7 \pm 0.06^{\mathrm{a}}$ & $2.0 \pm 0.09^{\mathrm{a}}$ & $2.2 \pm 0.08^{\mathrm{a}}$ & $2.5 \pm 0.07^{\mathrm{a}}$ & $2.9 \pm 0.06^{\mathrm{a}}$ \\
T1 & $1.3 \pm 0.03^{\mathrm{b}}$ & $2.0 \pm 0.06^{\mathrm{b}}$ & $2.4 \pm 0.09^{\mathrm{b}}$ & $2.7 \pm 0.08^{\mathrm{b}}$ & $3.1 \pm 0.07^{\mathrm{b}}$ & $3.8 \pm 0.06^{\mathrm{b}}$ \\
T2 & $1.5 \pm 0.03^{\mathrm{c}}$ & $2.5 \pm 0.06^{\mathrm{c}}$ & $3.0 \pm 0.09^{\mathrm{c}}$ & $3.2 \pm 0.08^{\mathrm{c}}$ & $3.6 \pm 0.07^{\mathrm{c}}$ & $4.2 \pm 0.06^{\mathrm{c}}$ \\
\hline$p$ & $<0.01$ & $<0.01$ & $<0.02$ & $<0.01$ & $<0.01$ & $<0.01$ \\
\hline
\end{tabular}

T0: Dieta comercial (DC); T1: DC con 10\% HM; T2: DC con $20 \% \mathrm{HM}$

Este desempeño posiblemente estuvo compensando por la energía metabolizable presente en la dieta, la cual se recomienda que debe tener un valor de $2750 \mathrm{kcal} / \mathrm{kg}$ cuando se utiliza una dieta única durante el crecimiento completo (Santomá, 1989), o valores de $2900 \mathrm{kcal} / \mathrm{kg}$ hasta los 2-3 semanas de edad (NRC, 1994; Lázaro et al., 2005) y de $3100-3200 \mathrm{kcal} / \mathrm{kg}$ hasta el beneficio (Vázquez, 2000; Lázaro et al., 2005). Considerando estas premisas, los niveles energéticos en las tres dietas cumplían estos estándares $(2600-3200 \mathrm{kcal} / \mathrm{kg})$.

La CA aumentó a medida que el porcentaje de HM fue mayor ( $<<0.01$; Cuadro 2). El efecto de la suplementación con HM sobre la CA ha sido también descrito en pollos de engorde, donde se evidenció la misma tendencia, pues a medida que se incluían mayores niveles de morera se desmerecía la CA (Casamachin, 2007; Simol, 2012; Leyva, 2012), ya que esta es afectada por el consumo de alimento y por la calidad de la dieta.

El consumo de alimento semanal fue significativamente diferente entre tratamientos, siendo mayor en las aves suplementadas con HM en comparación con las aves de T0 (Cuadro 3). Esto explicaría que los grupos suplementados con HM tuvieran una CA semanal acumulada mayor que las del grupo T0 (Cuadro 4). Aparentemente, un mayor porcentaje de fibra en la dieta (1.9 veces más en $\mathrm{T} 1$ y 2.4 veces más en $\mathrm{T} 2$ con respecto a T0) incrementó la tasa de pasaje de alimento en el tracto gastrointestinal, y con ello estimuló el consumo de alimento e incremento la 


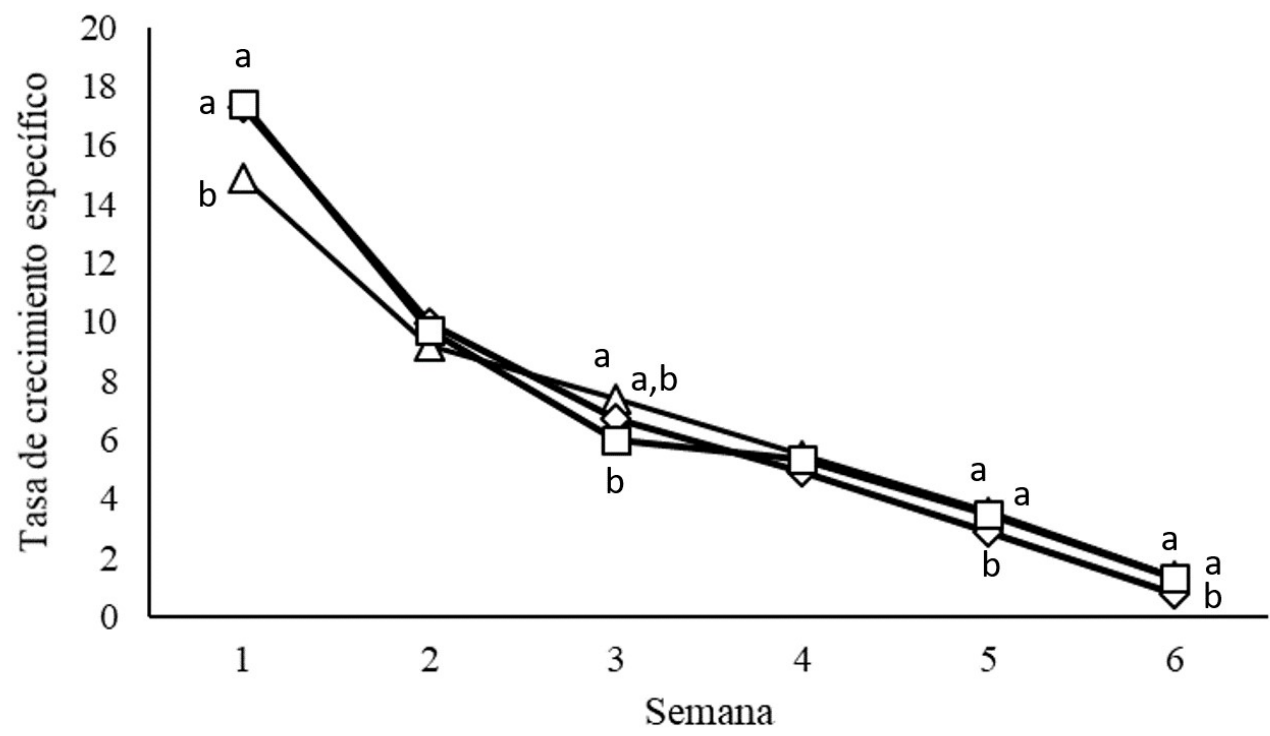

Figura 2. Tasa de crecimiento específico de codornices (Coturnix coturnix japonica) suplementadas con harina de morera (HM) durante 42 días. T0 (0\% HM): $\Delta$; T1 $(10 \% \mathrm{HM}): \checkmark ; \mathrm{T} 2(20 \% \mathrm{HM}): \boldsymbol{\square}$. Letras diferentes en la misma semana difieren ${ }^{\mathrm{a}, \mathrm{b}}$ $(\mathrm{p}<0.05)$

ganancia de peso en los grupos $\mathrm{T} 1$ y T2. Esto coincide con los hallazgos de la morfometría de órganos intestinales, cuyo análisis estadístico indicó que las aves de los grupos T1 y T2 tuvieron un ciego 2.3 y 2.8 veces más pesado que las de $\mathrm{T} 0$ ( $\mathrm{p}<0.01 ;$ datos no publicados).

Como es conocido, mientras menor es el valor de la CA es mayor el aprovechamiento del alimento consumido y, por lo tanto, mayor es la eficiencia alimenticia. En esta especie, el aprovechamiento del contenido proteico de la dieta puede reducirse ligeramente a partir de los 14 o 28 días, dando como resultado que los índices de CA aumenten rápidamente con la edad, indistintamente de la dieta utilizada (Lázaro et al., 2005). En este estudio, la CA aumentó proporcionalmente más en los grupos $\mathrm{T} 1$ y T2 que en T0 durante las seis semanas (Cuadro 4).

Es importante destacar que las aves de los tratamientos que recibieron HM presentaron heces con menor consistencia durante las tres primeras semanas, lo cual puede indicar que se aumentó la velocidad de pasaje del alimento por el tracto digestivo, probablemente por el mayor contenido de fibra que en el alimento comercial. Este fenómeno debe haber estimulado un consumo mayor de alimento, lo cual también determinó que estos grupos tuvieran una tasa de crecimiento mayor.

El análisis de la TCE indicó diferencias estadísticas entre T0 $(7.01 \%)$ y los grupos T1 $(7.13 \%)$ у T2 $(7.14 \%)(\mathrm{p}<0.05)$. A pesar de ser un parámetro no comúnmente evaluado en el crecimiento de especies terrestres, permite identificar las variaciones porcentuales del crecimiento animal (Caravaca et al., 2005), así como la intensidad y ritmo de crecimiento en las distintas fases de la vida del animal. La TCE es resultante de los incrementos en el consumo de alimento, así como de la eficiencia alimenticia.

Como se aprecia en la Figura 2, el crecimiento porcentual diario inicial de las aves- 
fue significativamente inferior en $\mathrm{T} 0$ que en T1 y T2. A partir de este momento y hasta la $3^{\text {ra }}$ semana, el ritmo de crecimiento disminuyó significativamente más en las aves que consumieron HM que en las alimentadas únicamente con $\mathrm{AB}$, siendo en este momento el valor de T0 similar a T1 y estadísticamente superior a T2. Entre la $3^{\text {ra }}$ y $4^{\text {ta }}$ semana, la tasa de crecimiento disminuyó a un ritmo similar y más intenso en T0 y T1 que en T2. De la $4^{\text {ta }}$ semana en adelante, la tasa de crecimiento decreció a un ritmo similar en los tres tratamientos, aunque esta fue significativamente superior en T0 que en $\mathrm{T} 1$. El análisis de la TCE demostró que el suministro de proteína en las dietas evaluadas (alrededor de $17 \%$, ) fue adecuada para que las aves experimentaran un crecimiento rápido y semejante.

El RC fue estadísticamente similar entre los tres tratamientos (Cuadro 2). El RC de los grupos evaluados se mantuvo dentro del rango de 65 y 77\% indicado por Santomá (1989). Otros estudios tampoco han reportado diferencias en el RC, indistintamente del nivel proteico evaluado (Kirkpinar y Oguz, 1995). La variación en el RC puede estar atribuida a las dietas suministradas (Díaz et al., 2009), técnicas de manejo y pesaje de los animales (Cori et al., 2009), así como también a las líneas genéticas utilizadas, que pueden determinar diferencias que oscilan entre un 10 y 20\% (Lázaro et al., 2005).

Se ha señalado que es conveniente beneficiar a las codornices a los 42-45 días (Cori et al., 2009; Díaz et al., 2007), ya que durante este periodo las codornices han alcanzado el peso adecuado, y a partir de esta edad, no experimentan cambios significativos en los pesos y rendimientos cárnicos, sino que más bien es el momento en que suele manifestarse la pubertad, y cuando otros parámetros como el PF y CA tienden a desmejorar. En este sentido las aves evaluadas en el presente ensayo mostraron pesos similares a los obtenidos por Cori et al. (2009), inclusive la dieta T0 (alimento comercial) se mostró más cercana a la reportada por estos autores, quienes señalaron un RC del 76.7\%.

La mortalidad durante las seis semanas de estudio no experimentó diferencias significativas entre tratamientos. Para la especie C. coturnix japonica, la mortalidad durante el periodo de crecimiento puede variar entre el 2 y $8 \%$ (Lázaro et al., 2005). Simol (2012) reportó una mayor mortalidad en pollos que recibieron dietas con más de $40 \%$ de $\mathrm{HM}$, debido a la baja aceptación de las dietas. Es importante indicar que la HM no contiene sustancias tóxicas que pudieran afectar el funcionamiento normal del ave (Casamachin et al., 2007; Leyva et al., 2012; Camacaro et al., 2015).

La relación $\mathrm{CB}$ fue mejor en las aves de T2, seguido por el T1 y T0 (Cuadro 2). Casamachin et al. (2007), asimismo, demostraron en pollos de engorde, la ventaja económica de incluir 5\% de HM. Este estudio demuestra que la utilización de HM puede reducir el costo de adquisición de alimentos comerciales, haciendo su utilización como ración alimenticia económica y productivamente factible.

\section{Conclusiones}

- La utilización de harina de morera (Morus alba) en codornices suplementadas durante seis semanas favoreció el desarrollo de las aves, principalmente entre la $2^{\text {da }}$ y $3^{\text {era }}$ semanas del periodo de evaluación.

- Los grupos suplementados con harina de morera obtuvieron una mejor repuesta productiva en términos de peso final y ganancia diaria de peso en comparación con las aves alimentadas solo con alimento comercial.

- La inclusión del 10\% de harina de morera fue una alternativa viable para favorecer el crecimiento de la $C$. coturnix japonica, y para reducir los costos de alimentación. 


\section{Agradecimiento}

Los autores expresan su agradecimiento al Consejo de Desarrollo Científico, Humanístico, Tecnológico y de las Artes (CDCHTA) de la Universidad de Los Andes, Venezuela, por el financiamiento de esta investigación (Proyecto NURR-C-559-12-01F). Igualmente se expresa agradecimiento al propietario de la Finca Dorana por suministrar la morera usada en este estudio.

\section{Literatura Citada}

1. Arslan C, Inal F, Garip M. 2000. The possibility of using full fat sunflower seed in Japanese quail diets. Veteriner Bilimleri Dergisi 16: 137-143.

2. [AOAC] Association of Official Analytical Chemists. 1995. Official methods of analysis. $16^{\text {th }}$ ed. Washington DC, USA: AOAC. $1230 \mathrm{p}$.

3. Bayram Y, Akinsi Z. 2001. The effect of the rations containing different amounts of ground nut meal on egg production and hatching results in laying quails. Ankara Univ Vet Fak 48: 35-41. doi: 10.1501/Vetfak 0000000233

4. Benavides J. 200 2. Utilization of mulberry in animal production systems. FAO Electronic Conference on mulberry for animal production (Morus 1-L). [Internet]. Available in: http://www.fao.org/livestock/agap/frg/mulberry/Papers/ PDF/Benavid.pdf

5. Camacaro L, Fernández D, Viveros M, Fernández E, Suárez B, Aranguren E, Salón A, et al. 2015. Producción de huevos de gallinas ponedoras Isa Brown en etapa de alta postura alimentadas con morera (Morus alba). Rev Fac Agron (UCV) 41: 35.

6. Caravaca F, Castel J, Guzmán J, Delgado M, Mena Y, Alcalde M, González P. 2005. Bases de la producción animal. España: Univ. de Sevilla. 512 p.
7. Casamachin ML, Ortiz D, López F. 2007. Evaluación de tres niveles de inclusión de morera (Morus alba) en alimento para pollos de engorde. Fac Cienc Agropec 5: 64-71.

8. Cori ME, De Basilio V, Figueroa $R$, Michelangeli C, Galíndez, R, García J. 2009. Efecto de la edad de la codorniz (Coturnix coturnix japonica) y del aturdimiento eléctrico al momento del beneficio sobre las características de la canal. Zoot Trop 27: 175-185.

9. Dalmau A. 1994. Manual de la codorniz: cría industrial y para la caza. España: Ed Dilagro. $67 \mathrm{p}$.

10. Díaz D, Briceño $R$, Cabrera $H$, González D. 2007. Factibilidad y edad de engorde en codornices (Coturnix coturnix japonica) suplementadas con harina de lombriz (Eisenia fetida). Agricultura Andina 15: 460-461.

11. Díaz D, Juárez E, Maffei M, Morón O, González L Morales J. 2009. Alimentación de codornices de engorde (Coturnix coturnix japonica) a base de harina de lombriz en dos niveles proteicos. Agricultura Andina 17:3-18.

12. Gaceta Oficial de Venezuela. 2000. Resolución Conjunta del Ministerio de Producción y Comercio y del Ministerio de Salud y Desarrollo Social. Gaceta ${ }^{\circ}$ 37.035

13. Genchev A, Mihaylova G, Ribarski S, Pavlov A, Kabakchiev M. 2008. Meat quality and composition in Japanese quails. Trakia J Sci 6: 72-82.

14. Hodgkinson S, Rosales E, Alomar D, Boroschek D. 2004. Evaluación químico-nutricional de alimentos secos comerciales en Chile para perros adultos en manutención. Arch Med Vet 36: 173-181. doi:10.4067/S0301-732X2004000-200008

15. Islam MR, Siddiqui MN, Khatun A, Siddiky MN, Rahman MZ, Bostami AB, Selim AS. 2014. Dietary effect of mulberry leaf (Morus alba) meal on growth performance and serum cholesterol level of broiler chickens. SAARC J Agric 12: 79-89. doi: 10.3329/sja.v12i2.21920 
16. Itzá MF, Lara PE, Magaña MA, Sanginés JR. 2010. Evaluación de la harina de hoja de morera (Morus alba) en la alimentación de pollos de engorda. Zoot Trop 28: 477-487.

17. Kirkpinar K, Oguz I. 1995. Influence of various dietary protein levels on carcase composition in the male Japanese quail (Coturnix coturnix japonica). Brit Poultry Sci 36: 605-610. doi: 10.1080/00071669508417806

18. Lázaro R, Serrano MP, Capdevila J. 2005. Nutrición y alimentación de avicultura complementaria: codornices. En: Federación Española para el Desarrollo de la Nutrición Animal. XXI Curso de Especialización FEDNA. Avances en Nutrición y Alimentación. Barcelona, España.

19. Leyva L, Olmo C, León E. 2012. Inclusión de harina deshidratada de follaje de morera (Morus alba L) en la alimentación del pollo campero. Rev Cient UDO Agríc 12: 653-659.

20. Martínez M, Motta W, Cervera C, Pla M. 2005. Feeding mulberry leaves to fattening rabbits: effects on growth, carcass characteristics and meat quality. Anim Sci 80: 275-281. doi: 10.1079/ ASC41110275

21. Morantes M. 1995. Efectos del uso del aceite crudo de la palma africana (Elaeis guinneensis) sobre el comportamiento productivo, contenido de colesterol sanguíneo y lípidos corporales totales de la codorniz (Coturnix coturnix japonica). Tesis de Grado. Maracay, Venezuela: Univ. Central de Venezuela. 82 p.

22. Nieves D, Cordero J, Terán O, González C. 2004. Aceptabilidad de dietas con niveles crecientes de morera (Morus alba) en conejos destetados. Zoot Trop 22: 202-209.

23. [NRC] National Research Council. 1994. Nutrient requirements of poultry. $9^{\text {th }}$ ed. Washington, USA: NRC.

24. Panja P. 2013. The effects of dietary mulberry leaves (Morus alba L) on chicken performance, carcass, egg quality and cholesterol content of meat and egg. Walailak J Sci Tech 10: 121129. doi: 10.2004/wjst.v10i2.306

25. Real F. 1996. Efectos de la sustitución de la harina de maíz Zea mays por harina de batata (Ipomoea batatas L Lam) como fuente energética sobre el comportamiento productivo de la codorniz (Coturnix coturnix japonica) en fase de postura. Tesis de Grado. Maracay, Venezuela: Univ. Central de Venezuela. 78 p.

26. Santomá G 1989. Nutrition of domestic quails. Proc $7^{\text {th }}$ European Symposium of Poultry Nutrition. Barcelona, España: Word's Poultry Science Association.

27. [SAS] Statistical Analysis Systems Institute. 2012. User's Guide. University North of Caroline, USA. Version 9.2.

28. Segura JC, Honhold N. 2000. Métodos de muestreo para la producción y la salud animal. Yucatán, México: Univ. Autónoma de Yucatán. $142 \mathrm{p}$.

29. Simol CF, Tuen AA, Ahmad A, Chubo JK, King PJ, Ong KH. 2012. Performance of chicken broilers fed with diets substituted with mulberry leaf powder. Afr J Biotechnol 11: 16106-16111. doi: 10.5897/AJB12.1622

30. Sulca P, Figueroa E, Carcelén F. 2000. Evaluación de los parámetros productivos de codornices variedad japonesa (Coturnix coturnix japonica) provenientes de tres planteles reproductores de Lima. Rev Inv Vet Perú 11: 153-162. doi: 10.15381/rivep.v11i2.7354

31. Ustundag AO, Ozdogan M. 2015. Usage possibilities of mulberry leaves in poultry nutrition. Scientific Papers, Series D. Anim Sci 58: 170-178.

32. Vatsalya V, Arora K. 2011. Association between body weight growth and selected physiological parameters in male japanese quail (Coturnix japonica). Int J Poult Sci 10: 680-684. doi: 10.3923/ ijps.2011.680.684

33. Vázquez R, Ballesteros H. 2000. La cría de codornices. Colombia: Produmedios Ediciones. 68 p. 